\title{
Estabilidade oxidativa do colesterol em ovo integral em pó
}

\author{
Claudia Escarabajal, Alfredo Tenuta Filho*
}

Departamento de Alimentos e Nutrição Experimental, Faculdade de Ciências Farmacêuticas,

Universidade de São Paulo

*Correspondência:

A. Tenuta-Filho

Faculdade de Ciências Farmacêuticas.

USP

Departamento de Alimentos e Nutrição

Experimental

Av. Prof. Lineu Prestes, 580, Bloco 14,

Butantã.

05508-900 - São Paulo-SP-Brasil

E-mail: eetenuta@usp.br
Ovos são importantes como alimento e como matéria-prima industrial. Ao mesmo tempo, têm elevada concentração de colesterol, principalmente quando desidratado. O colesterol, por sua vez, está sujeito à oxidação durante o processamento elou estocagem, formando, em conseqüência, derivados oxidados com atividades tóxicas, entre as quais a aterogenicidade. Foi avaliada, neste trabalho, a estabilidade do colesterol em ovo integral em pó comercial, através da ocorrência do 7-cetocolesterol livre, quantificado por cromatografia líqüida de alta eficiência, depois da extração dos lípides totais e separação em coluna de Florisil. O 7-cetocolesterol livre ocorreu em todos os três lotes das cinco marcas analisadas de ovo integral em pó, em teor médio de 84,01 55,34 $\mathrm{Pg} / \mathrm{g}$ de lípides. Não foi possível indicar clara evolução da oxidação do colesterol durante a estocagem do produto por até 224 dias, à temperatura ambiente; no entanto, uma correlação significativa $(r=0,8093)$ pôde ser observada entre o 7cetocolesterol livre e as substâncias reativas ao ácido tiobarbitúrico (TBARS).

\section{INTRODUÇÃO}

Estudos sobre a oxidação do colesterol em alimentos têm sido motivados principalmente pelas características biológicas dos óxidos formados, interferindo na morfologia e função da membrana celular, inibindo a biossíntese do próprio colesterol e sendo aterogênicos (Brown, Jessup, 1999; Peng, Hu, Morin,1991), citotóxicos (Osada, 2002; Ohtani et al., 1996; 1997; Sevanian, Peterson, 1986; Smith, Johnson, 1989), mutagênicos e carcinogênicos (Guardiola et al., 1996; Sevanian, Peterson, 1986).

Muitos óxidos de colesterol (OsC) têm sido identifi- cados (Tai et al., 1999), mas somente oito deles são os mais freqüentemente encontrados em alimentos: 7 cetocolesterol (7-ceto), 20-hidroxicolesterol (20-OH), 25hidroxicolesterol (25-OH), 7D-hidroxicolesterol (7D-OH), 7E-hidroxicolesterol (7E-OH), colesterol-5,6D-epóxido (5,6D-epóxido), colesterol-5,6E-epóxido (5,6E-epóxido) e colestanotriol (Triol) (Addis et al., 1996; Lercker, Rodriguez-Estrada, 2000; Pie, Spahis, Seillan, 1990; Tai, Chen, Chen, 1999). O 7-ceto tem sido proposto como indicador da oxidação do colesterol, por ser o de maior ocorrência em muitos alimentos.

Em geral, considera-se que em alimentos frescos óxidos de colesterol não são detectáveis (Nourooz-Zadeh, 
Appelqvist, 1987; Sarantinos, O’Dea, Sinclair, 1993), e que sua formação pode se dar durante o processamento (doméstico inclusive) e/ou estocagem do alimento (Huber, Pike, Huber, 1995; Lai et al., 1995; Sander et al., 1989), motivada por fatores tais como o oxigênio atmosférico, radiação, temperatura, radicais livres, íons metálicos, tempo e temperatura de armazenamento (Maerker, 1987; Paniangvait et al., 1995; Smith, 1987; Tai, Chen, Chen, 2000).

O ovo é um importante alimento e também matériaprima industrial. Seu conteúdo em colesterol, cerca de 400 $\mathrm{mg} / 100 \mathrm{~g}$, é um dos mais elevados entre os alimentos (aproximadamente duas vezes mais que o da manteiga e cinco vezes maior que o da carne) (Galobart, Guardiola, 2002).

O processo de obtenção do ovo integral em pó ou gema em pó é feito por atomização, concentrando-se os teores de colesterol por desidratação em 929 a 1918 mg/100 g (Pie et al., 1990; Sarantinos, O'Dea, Sinclair, 1993). Diversos estudos têm relatado a formação de $\mathrm{OsC}$ em ovo integral em pó obtido por atomização, relacionada com o tipo do equipamento e temperatura do processo, além das condições de estocagem (Guardiola et al.,1997; Huber, Pike, Huber, 1995; Lai et al., 1995; Sander et al., 1989; Sarantinos, O'Dea, Sinclair, 1993; Zunin et al., 1995).

Neste trabalho foi avaliada a estabilidade do colesterol, medida pela ocorrência do 7-cetocolesterol livre, em amostras comerciais de ovo integral em pó e verificada a possível formação desse óxido durante a estocagem do produto.

\section{MATERIAL E MÉTODOS}

\section{Material}

Isopropanol e $n$-hexano utilizados na cromatografia líqüida de alta eficiência tinham grau HPLC e os demais reagentes grau p.a. 6-Cetocolestanol, 7-cetocolesterol e colesterol foram obtidos da Sigma e o Florisil (MgO:SiO2, 15:85), da Merck.

As amostras comerciais de ovo integral em pó corresponderam a três lotes de 5 marcas (A, B, C, D e E), cedidas por produtores e/ou fornecedores, e analisadas entre 15 a 120 dias após a data de fabricação, portanto, dentro do prazo de validade de 180 dias. Detalhes operacionais do processo industrial de atomização das amostras não foram fornecidos.

\section{Umidade, lípides totais e substâncias reativas ao ácido tiobarbitúrico (TBARS)}

A umidade foi analisada com base na perda de substâncias voláteis a $105^{\circ} \mathrm{C}$ (Instituto Adolfo Lutz, 1985); os lípides totais, por extração com clorofórmio/metanol (2:1, v/v) (Folch, Lees, Stanley, 1957), evaporação do extrato em rotoevaporador $\left(40{ }^{\circ} \mathrm{C}\right)$ sob vácuo, e pesagem do resíduo obtido; e TBARS (substâncias reativas ao ácido tiobarbitúrico) pelo método de Vyncke (1970), empregando-se o antioxidante BHT (butil-hidroxitolueno) a 0,02\% em relação aos lípides totais.

\section{Colesterol}

A extração lipídica seguiu o proposto por Folch, Lees, Stanley (1957) e o extrato lipídico foi evaporado e submetido à saponificação a frio $\left(25 \mathrm{a} 30^{\circ} \mathrm{C}\right)$, por 18 horas, com $20 \mathrm{~mL}$ de $\mathrm{KOH} 1 \mathrm{M}$, em metanol, conforme Chen, Chen (1994). A fração insaponificável foi extraída com $10 \mathrm{~mL}$ de $n$-hexano, por três vezes consecutivas e o solvente evaporado a $40{ }^{\circ} \mathrm{C}$, em rotoevaporador, sob vácuo. $\mathrm{O}$ resíduo gerado, usado na quantificação do colesterol, segundo Csallany et al. (1989), depois de ressuspenso em $2 \mathrm{~mL}$ da fase móvel $n$-hexano/isopropanol, 97:3, foi filtrado em membrana durapore (Millex-Millipore), com 0,45 Pm de diâmetro, e automaticamente injetado (alíquotas de 20 PL) no HPLC Shimadzu LC-10ADVP. Foi usada coluna P-Porasil, com $10 \mathrm{Pm}$ de poro, de 30 x $0,39 \mathrm{~cm}$ (Waters Associates), em fase normal, com o fluxo de $1 \mathrm{~mL} / \mathrm{min}$. O monitoramento se deu a $206 \mathrm{~nm}$ utilizando-se detector de fotodiodos. Os parâmetros de validação do citado método constam em Morales-Aizpurúa, Tenuta-Filho (2005).

\section{7-Cetocolesterol livre}

A extração lipídica foi feita conforme descrita por Folch, Lees, Stanley (1957), com adição prévia de 6-cetocolestanol (22 a 24 Pg), como padrão interno (Li, Cherian, Sim, 1996; Chen, Chen ,1994; Maerker, Unruh, 1986), e de BHT, a 0,02 $\%$, calculado em relação aos lípides (Du, Ahn, 2000). A separação em fase sólida consistiu do procedimento (método $\mathrm{A}$ ) empregado por Penazzi et al. (1995): o extrato lipídico (50-60 $\mathrm{mg}$ ) foi diluído com 0,5 mL de $n$-heptano / isopropanol (98:2, $\mathrm{v} / \mathrm{v}$ ), adicionado em coluna de Florisil (500 mg) e eluído com acetona. Após a evaporação da acetona, em rotoevaporador a $40^{\circ} \mathrm{C}$, sob vácuo, o resíduo foi dissolvido em $2 \mathrm{~mL}$ da fase móvel n-hexano/iso-propanol (93:7 v/v), filtrado em membrana durapore (Millex-Millipore), e automaticamente injetado (20 PL) no cromatógrafo, como no caso do colesterol (Csallany et al.,1989), com monitoramento a $233 \mathrm{~nm}$. A validação do método foi feita com base na recuperação $(n=5)$ do 7-cetocolesterol $(99,06 \pm 0,00 \%)$ e do 6-cetocolestanol $(94,82 \pm 0,03 \%)$, usado como padrão interno, adicionados ao ovo integral em pó $(0,1 \mathrm{~g})$ antes da extração com clorofórmio/ metanol. 


\section{Estabilidade do colesterol durante a estocagem}

Foram usados três lotes distintos de ovo integral em pó (marca E), obtidos entre 24 e 30/04/2003. Cada lote foi homogeneizado, subdividido em porções de $100 \mathrm{~g}$ cada, reembalado em sacos plásticos (polipropileno), lacrados com auxílio de fita adesiva, depois de prévia retirada parcial do ar (expulsão por pressionamento manual). As amostras ficaram estocadas por até 224 dias, à temperatura ambiente, e foram analisadas a cada 30 dias até o $180^{\circ}$ dia. A temperatura variou entre 12 a $29^{\circ} \mathrm{C}$. As amostras foram expostas à mesma intensidade de luz, de tal forma que a parte superior das mesmas ficasse voltada para baixo, a cada três dias. O período inicial do experimento correspondeu ao uso de amostras com 7-15 dias de fabricação.

\section{Análise estatística}

Os dados obtidos foram submetidos à análise de variância (Anova), segundo o programa Instat versão 2.01 (Graph Pad Software). O coeficiente de correlação linear de Pearson foi empregado segundo Costa-Neto (1977).

\section{RESULTADOS E DISCUSSÃO}

\section{Colesterol e 7-cetocolesterol livre em ovo integral em pó}

As amostras comerciais de ovo integral em pó analisadas apresentaram conteúdo médio de colesterol de $1.111,7 \pm 11,4 \mathrm{mg} / 100 \mathrm{~g}$ (Tabela I). Os resultados obtidos confirmam o fato de o produto ser fonte de colesterol em elevada concentração, ao mesmo tempo que são concordantes com os verificados por Sarantinos, O'Dea, Sinclair (1993), 928,9-1.110,3 mg/100 g, e inferiores aos relatados por Pie et al. (1990), 1.330-1.410 mg/100 g.

TABELA I - Colesterol em ovo integral em pó

\begin{tabular}{lc}
\hline Concentração (base seca) & Colesterol (mg/100 g) \\
\hline Mínima & 991,4 \\
Máxima & $1.137,6$ \\
Média (n=3) & $1.111,7$ \\
Desvio-padrão $( \pm)$ & 11,4 \\
Coeficiente de variação (\%) & 1,0 \\
\hline
\end{tabular}

Nos três lotes distintos de cada uma das cinco marcas de ovo integral em pó (A-E), com 15 a 120 dias de fabricação, foram encontrados teores médios de umidade variáveis, considerando os coeficientes de variação constatados. O valor médio geral relativo às marcas, de $2,26 \pm 0,54 \mathrm{~g} / 100 \mathrm{~g}$ (Tabela II), ficou situado abaixo do valor máximo declarado pelos fabricantes $(\mathrm{d} 4 \mathrm{~g} / 100 \mathrm{~g})$. Os resultados de umidade obtidos são relativamente baixos e contribuem para a estabilidade química e microbiológica do produto.

Conforme a Tabela II, foi detectado um valor médio de 7-cetocolesterol livre de 84,01 $\pm 5,34 \mathrm{Pg} / \mathrm{g}$ de lípides para as marcas de ovo integral em pó estudadas, ou seja, cerca de $0,29 \%$ do colesterol (Tabela I). Certamente este

TABELA II - Umidade, 7-cetocolesterol livre, TBARS e lípides totais (base seca) em três lotes diferentes de cinco marcas comerciais de ovo integral em pó

\begin{tabular}{ccccc}
\hline $\begin{array}{c}\text { Marcas } \\
(\mathbf{M} \pm \mathbf{D P})^{*}\end{array}$ & $\begin{array}{c}\text { Umidade } \\
(\mathbf{g} / \mathbf{1 0 0} \mathbf{g})\end{array}$ & $\begin{array}{c}\text { 7-cetocolesterol livre } \\
(\boldsymbol{\mu} \mathbf{g} / \mathbf{g} \text { de lípides })\end{array}$ & $\begin{array}{c}\text { TBARS } \\
(\mathbf{m g ~ D A M} / \mathbf{k g})\end{array}$ & $\begin{array}{c}\text { Lípides totais } \\
(\mathbf{g} / \mathbf{1 0 0} \mathbf{g})\end{array}$ \\
\hline $\mathrm{A}$ & $2,27 \pm 0,89$ & $85,98 \pm 9,15$ & $1,17 \pm 0,04$ & $38,53 \pm 0,54$ \\
& $(38,93)$ & $(10,65)$ & $(3,44)$ & $(1,40)$ \\
$\mathrm{B}$ & $2,73 \pm 0,70$ & $90,41 \pm 20,15$ & $1,09 \pm 0,06$ & $37,45 \pm 0,14$ \\
& $(25,55)$ & $(22,29)$ & $(5,11)$ & $(0,42)$ \\
$\mathrm{C}$ & $1,34 \pm 0,27$ & $75,96 \pm 2,52$ & $1,31 \pm 0,19$ & $37,80 \pm 1,04$ \\
& $(19,73)$ & $(3,31)$ & $(141,15)$ & $(2,74)$ \\
$\mathrm{D}$ & $2,57 \pm 0,86$ & $85,36 \pm 17,14$ & $0,76 \pm 0,04$ & $40,89 \pm 1,25$ \\
& $(33,63)$ & $(20,08)$ & $(5,45)$ & $(3,07)$ \\
$\mathrm{E}$ & $2,37 \pm 0,56$ & $82,35 \pm 24,18$ & $0,83 \pm 0,141$ & $34,12 \pm 3,78$ \\
& $(23,78)$ & $(29,36)$ & $(16,74)$ & $(11,08)$ \\
\hline $\mathrm{M} \pm \mathrm{DP} * *$ & $2,26 \pm 0,54$ & $84,01 \pm 5,34$ & $1,03 \pm 0,23$ & $37,76 \pm 2,43$ \\
$(\mathrm{CV})$ & $(23,95)$ & $(6,36)$ & $(22,45)$ & $(6,45)$ \\
\hline
\end{tabular}

* Média \pm Desvio-padrão (n=3); DAM = Dialdeído malônico; ** Média \pm Desvio-padrão para as marcas A-E (n=5); $\mathrm{CV}=$ Coeficiente de variação, \%. 
grau de oxidação do colesterol está subestimado. Isso porque outros óxidos que também estariam presentes no produto, conforme descrito na literatura, não foram quantificados neste trabalho. O 7-cetocolesterol livre pode ter sido formado durante o processo de atomização e/ou na estocagem subseqüente a que o produto foi submetido.

Os conteúdos de 7-cetocolesterol livre indicados na Tabela II, na maioria dos casos, foram maiores que os relatados por outros autores, inclusive os relativos ao 7cetocolesterol total, conforme a Tabela III. Segundo Penazzi et al. (1995), a maioria do 7-cetocolesterol presente no ovo está na forma livre não-esterificada.

As TBARS foram quantificadas num valor médio de $1,03 \pm 0,23 \mathrm{mg}$ de DAM/kg de amostra (Tabela II), indicando ter havido oxidação de ácidos graxos. Os fatores que promoveram ou facilitaram a oxidação dos ácidos graxos certamente contribuíram para a do colesterol, expressada pelo 7-cetocolesterol livre quantificado (Tabela II). De acordo com a Tabela II, a presença de lípides no ovo integral em pó é bastante significativa, em valor médio de 37,76 $\pm 2,43$ $\mathrm{g} / 100 \mathrm{~g}$. Igualmente é a insaturação dos ácidos graxos desta fração lipídica. Cerca de $65 \%$ de ácidos graxos insaturados [principalmente palmitoléico (C16-1), 3,9\%; oléico (C18:1), 44,7 \%; linoléico (C18:2), 11,6\%] compõem a fração lipídica do ovo (Jiang, Sim, 1994). Entretanto, nenhuma correlação foi encontrada entre os níveis de 7-cetocolesterol livre e os das TBARS nas amostras analisadas na Tabela II.

\section{Estabilidade do colesterol durante a estocagem do ovo integral em pó}

Durante a estocagem de até 224 dias, à temperatura ambiente $\left(12-29^{\circ} \mathrm{C}\right)$, a umidade do ovo integral em pó apresentou aumento discreto, de 2,48 a 6,16 g/100 g (Tabela IV), não impedido pelas condições de embalagem plástica (polipropileno) usada. Nas amostras do produto com 15-120 dias de fabricação, analisadas anteriormente, a umidade não ultrapassou 2,26 $\pm 0,54 \mathrm{~g} / 100 \mathrm{~g}$ (Tabela II), abaixo do máximo indicado pelos fabricantes $(\mathrm{d} 4 \mathrm{~g} / 100 \mathrm{~g})$. Ao contrário da umidade, os lípides não variaram.
Os resultados de 7-cetocolesterol livre não permitiram provar sua formação durante a estocagem considerada, como conseqüência da oxidação do colesterol. Entre o início do experimento e o $60^{\circ}$ dia de estocagem houve aparente redução nos níveis do citado óxido, sem que causa justificável pudesse ser apontada. A partir do $90^{\circ}$ dia, os resultados poderiam estar indicando aumento até o $150^{\circ}$ dia, para depois, então, registrar aparente diminuição.

A evolução das TBARS durante o experimento, expressando tendência de aumento consistente e indicando a oxidação de ácidos graxos (Tabela IV; Figura 1), foi o suficiente para estabelecer correlação com o 7cetocolesterol livre [coeficiente de correlação: $r=0,8093$ $(\mathrm{p}<0,01)$; equação da reta: $\mathrm{y}=0,0483 \mathrm{x}-2,7098]$.

A tendência de redução na formação do 7 cetocolesterol livre observada após o $150^{\circ}$ dia de estocagem (Tabela IV; Figura 1), eventualmente poderia ser explicada com base em Lai et al. (1995). Esses autores estudaram o ovo em pó, obtido por atomização com sistema de aquecimento indireto do ar (elétrico), embalado em bolsa plástica (polyethylene-laminated nylon), sob vácuo, avaliando os óxidos epóxidos ( $\mathrm{De} E)$ e os derivados do carbono 7 (C-7) (7D-OH, 7E-OH e 7-ceto). O total de óxidos no início foi de 8,57 Pg/g; aos 6 meses de estocagem, aumentou para $42,54 \mathrm{Pg} / \mathrm{g}$, cerca de cinco vezes mais. A participação dos óxidos do C-7, que no início do experimento correspondia a $65,5 \%$ do total, foi reduzida para 42,1\%; a dos epóxidos aumentou, de 34,5\% para $57,9 \%$.

A concentração média do 7-cetocolesterol livre verificada no início do experimento, em produtos com tempo de fabricação de 7 a 15 dias, correspondeu a 76,88 \pm $5,59 \mathrm{Pg} / \mathrm{g}$ de lípides (Tabela IV), menor que a da maioria das amostras da Tabela II. É bem provável, portanto, que a oxidação do colesterol registrada já no inicio do experimento tenha se dado mais acentuadamente durante o processamento.

Desta forma, a suspeita recai sobre o tipo de processo, pelos antecedentes conhecidos indicados na literatura, em razão de a matéria-prima, no caso o ovo, não apresentar

TABELA III - 7-cetocolesterol livre e total em ovo integral em pó

\begin{tabular}{ll}
\hline 7-cetocolesterol ( $\boldsymbol{\mu g} / \mathbf{g}$ de lípides) & Autores \\
\hline $75,96-90,41$ (livre) & Tabela II (neste trabalho) \\
$20,2-58,0$ (livre; método A) & Penazzi et al. (1995) \\
$22,2-61,8$ (livre; método B) & Penazzi et al. (1995) \\
$38,4-83,7$ (total) & Sarantinos, O'Dea, Sinclair (1993) \\
$30,2-44,1$ (total) & Pie, Spahis, Seillan et al. (1990) \\
$29,0-104,0$ (total) & Lercker, Rodriguez-Estrada (2000) \\
\hline
\end{tabular}


TABELA IV -Umidade, 7-cetocolesterol livre, TBARS e lípides totais em ovo integral em pó estocado à temperatura ambiente, em até 224 dias

\begin{tabular}{ccccc}
\hline $\begin{array}{c}\text { Tempo de } \\
\text { estocagem (dias) }\end{array}$ & $\begin{array}{c}\text { Umidade } \\
(\mathbf{g} / \mathbf{1 0 0} \mathbf{g})\end{array}$ & $\begin{array}{c}\text { 7-cetocolesterol livre } \\
\mathbf{( P g / g ~ l i ́ p i d e s ) ~}\end{array}$ & $\begin{array}{c}\text { TBARS } \\
(\mathbf{m g D A M} / \mathbf{k g})\end{array}$ & $\begin{array}{c}\text { Lípides totais } \\
(\mathbf{g} / \mathbf{1 0 0} \mathbf{g})\end{array}$ \\
\hline 0 & $2,48 \pm 0,20^{\mathrm{a}}$ & $76,88 \pm 5,59^{\mathrm{a}}$ & $0,37 \pm 0,15^{\mathrm{a}}$ & $31,81 \pm 1,10^{\mathrm{a}}$ \\
$(8,09)$ & $(7,28)$ & $(10,34)$ & $(3,48)$ \\
30 & $2,08 \pm 0,72^{\mathrm{a}}$ & $64,91 \pm 4,71^{\mathrm{a}}$ & $0,17 \pm 0,07^{\mathrm{b}}$ & $32,06 \pm 1,46^{\mathrm{a}}$ \\
& $(6,53)$ & $(7,25)$ & $(13,28)$ & $(4,56)$ \\
60 & $4,07 \pm 0,27^{\mathrm{b}}$ & $66,92 \pm 1,91^{\mathrm{a}}$ & $0,43 \pm 0,04^{\mathrm{a}}$ & $33,94 \pm 1,31^{\mathrm{a}}$ \\
& $(6,53)$ & $(2,84)$ & $(3,25)$ & $(3,85)$ \\
90 & $4,45 \pm 0,37^{\mathrm{b}}$ & $79,84 \pm 6,31^{\mathrm{a}, \mathrm{b}}$ & $1,23 \pm 0,41^{\mathrm{c}}$ & $33,45 \pm 1,00^{\mathrm{a}}$ \\
& $(8,40)$ & $(7,90)$ & $(10,79)$ & $(2,99)$ \\
120 & $4,21 \pm 0,46^{\mathrm{b}}$ & $89,41 \pm 6,46^{\mathrm{b}}$ & $1,40 \pm 0,45^{\mathrm{c}, \mathrm{d}}$ & $35,20 \pm 1,00^{\mathrm{a}}$ \\
& $(10,92)$ & $(7,23)$ & $(10,54)$ & $(2,83)$ \\
150 & $5,38 \pm 0,51^{\mathrm{c}}$ & $98,33 \pm 13,47^{\mathrm{b}}$ & $1,64 \pm 0,11^{\mathrm{d}}$ & $35,13 \pm 0,52^{\mathrm{a}}$ \\
& $(9,44)$ & $(13,70)$ & $(2,23)$ & $(1,49)$ \\
180 & $5,71 \pm 0,32^{\mathrm{c}}$ & $80,22 \pm 1,71^{\mathrm{b}}$ & $1,86 \pm 0,67^{\mathrm{d}}$ & $33,04 \pm 1,50^{\mathrm{a}}$ \\
& $(5,60)$ & $(2,13)$ & $(11,98)$ & $(4,54)$ \\
224 & $6,16 \pm 0,36^{\mathrm{c}}$ & $82,73 \pm 2,22^{\mathrm{b}}$ & $2,06 \pm 0,19^{\mathrm{d}}$ & $31,05 \pm 2,82^{\mathrm{a}}$ \\
& $(5,82)$ & $(2,68)$ & $(9,47)$ & $(9,09)$ \\
\hline
\end{tabular}

Média \pm Desvio-padrão ( $\mathrm{n}=3$ ); (Coeficiente de variação, \%); Letras superscritas iguais nas colunas indicam diferenças estatísticas não-significativas, ao contrário das diferentes $(\mathrm{p}<0,05)$.

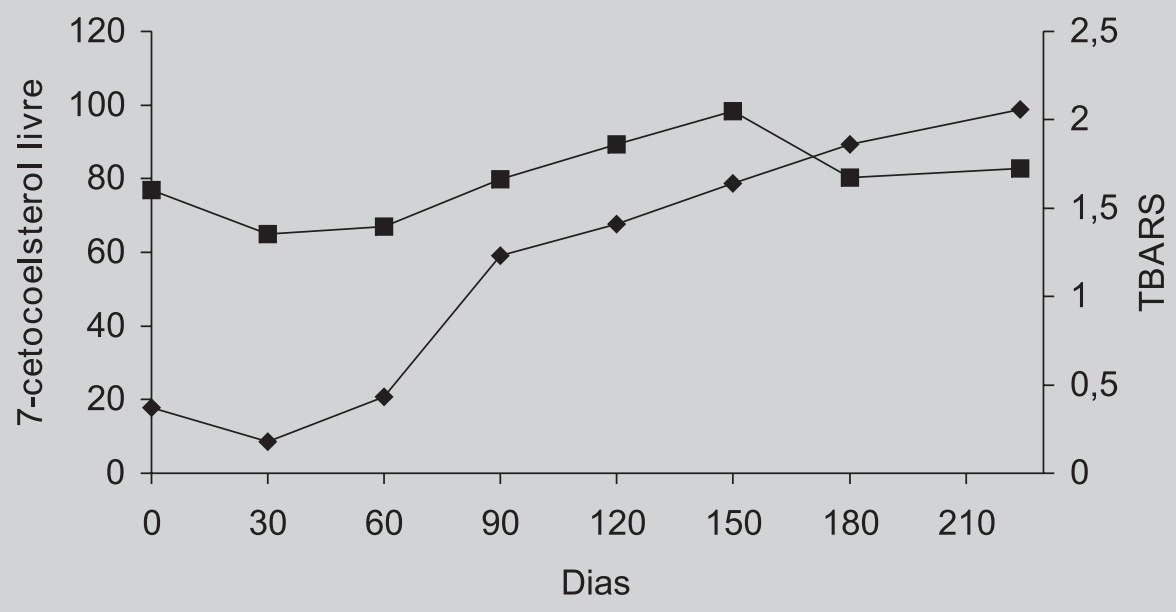

\section{7-cetocolesterol livre $\multimap$ TBARS}

FIGURA 1 - Evolução de 7-cetocolesterol livre (Pg/g de lípides) e TBARS (mg de DAM/kg) durante a estocagem do ovo integral em pó.

oxidação do colesterol estando no estado fresco (Sarantinos, O’Dea, Sinclair, 1993; Nourooz-Zadeh, Alppelqvist, 1987; Tai, Chen, Chen, 1999; Guardiola et al., 1994; Zunin et al., 1995). Em condições de aquecimento do ar com chama direta (gás), uma das formas empregadas na atomização do ovo (integral ou gema), tem sido apontada formação significativa de $\mathrm{OsC}$, em decorrência da produção de óxidos de nitrogênio $\left(\mathrm{NO}, \mathrm{NO}_{2}\right)$ gerados na combustão do gás, que podem atuar como iniciadores da autoxidação do colesterol (Lai et al., 1995; Tsai, Hudson, 1985; Morgan, Armstrong, 1992). Em condições de aquecimento indireto do ar (elétrico) no processo de 
atomização, foi verificado que a alteração das temperaturas (de entrada/saída) de $170 / 117^{\circ} \mathrm{C}$ para $225 / 140{ }^{\circ} \mathrm{C}$ promovia acréscimo na formação de OsC: $71,82 \%$ de Depóxido, 51,43\% de 7E-OH, 52,36\% de Triol, 35,27\% de 7 -ceto e $25,32 \%$ de $25-\mathrm{OH}$ (Guardiola et al., 1997).

O ovo desidratado (integral ou gema) ao compor a formulação de outros alimentos, como matéria-prima, poderá ser submetido a processos envolvendo aquecimento e outros fatores que favoreçam a oxidação adicional do colesterol. É importante então considerar o efeito que o processamento de fabricação de ovo em pó possa ter em relação à oxidação do colesterol, com o objetivo de tornar nula, ou reduzir a um mínimo inevitável, a formação de $\mathrm{OsC}$.

\section{CONCLUSÕES}

Foi constatada a presença de colesterol oxidado em ovo integral em pó comercial, detectada pela ocorrência de 7-cetocolesterol livre. Não foi possível indicar clara evolução da oxidação do colesterol durante a estocagem, mas uma correlação significativa pôde ser obtida entre o referido óxido e as TBARS, medidas como dialdeído malônico.

\section{ABSTRACT \\ Oxidative stability of cholesterol in whole egg powder}

Eggs are important as food and also as raw material for the food industry. However they show high levels of cholesterol, mainly when they are dehydrated. Cholesterol can be oxidized during processing and/or storage to cholesterol oxides which are toxics. The oxidative stability of cholesterol in commercial whole egg powder was evaluated by the occurrence of "free" 7-ketocholesterol (free 7-k) quantified by highperformance liquid chromatography, after total lipid extraction and separation on Florisil column. Free 7$k$ occurred in all samples analyzed (three different lots

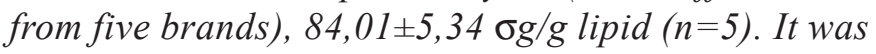
not possible to accompany cholesterol oxidation in whole egg powder stored until 224 days, at room temperature, but a significant correlation $(r=0.8093)$ was observed between free 7- $k$ and thiobarbituric acid reactive substances, attesting the actual oxidation of cholesterol to toxic keto derivative.

UNITERMS: Cholesterol. Cholesterol oxidation. $7-$ Ketocholesterol. Egg powder.

\section{AGRADECIMENTOS}

À CAPES, pela bolsa de estudo concedida à autora Escarabajal, C., e à FAPESP e ao CNPq, pela infra-estrutura de laboratório existente, decorrente de projetos de pesquisa que apoiaram.

\section{REFERÊNCIAS BIBLIOGRÁFICAS}

ADDIS, P.B.; PARK, P.W.; GUARDIOLA, F.; CODONY, R. Analysis and health effects of cholesterol oxides. In: McDONALD, R.E.; MIN, D.B.J., eds. Food lipids and health. New York: Marcel Dekker, 1996. p.99-240.

BROWN, O.; JESSUP, W. Oxysterols and atherosclerosis. Atherosclerosis, Shannon, v.142, p.1-28, 1999.

CHEN, B.H.; CHEN, Y.C. Evaluation of the analysis of cholesterol oxides by liquid chromatography. $J$. Cromatogr., A, Amsterdam, v.661, p.127-136, 1994.

COSTA NETO, P.L.O. Estatística. 1. ed. São Paulo: Ed. Edgard Bucher Ltda. 1977. 264p.

CSALLANY, A.S.; KINDOM, S.E.; ADDIS, P.B.; LEE, J. HPLC method for quantitation of cholesterol and four of its major oxidation products in muscle and liver tissues. Lipids, Champaign, v.24, n.7, p.645-651, 1989.

DU, M.; AHN, D.U. Effects of antioxidants and packaging on lipid and cholesterol oxidation and color changes of irradiated egg yolk powder. J. Food Sci., Chicago, v.65, n.4, p.625-629, 2000.

FOLCH, J.; LEES, M.; STANLEY, G.H.S. A simple method for the isolation and purification of total lipids from animal tissues. J. Biol. Chem., Baltimore, v.726, p. 497-509, 1957.

GALOBART, J.; GUARDIOLA, F. Formation and content of cholesterol oxidation products in egg and egg products. In: GUARDIOLA, F.; DUTTA, P.C.; CODONY, R.; SAVAGE, G.P. eds. Cholesterol and phytosterol oxidation products: analysis, occurrence, and biological effects. Champaign: AOCS Press, 2002. p. 124-146.

GUARDIOLA, F.; CODONY, R.; ADDIS, P.B; RAFECAS, M.; BOATELLA, J. Biological effects of oxysterols: current status. Food Chem. Toxic., Oxford, v.34, n.2, p.193-211, 1996. 
GUARDIOLA, F.; CODONY, R.; RAFECAS, M.; BOATELLA, J. Formación de derivados oxidados del colesterol en alimentos. Graxas Aceites, Sevilla, v.45, n.3, p.164-192, 1994.

GUARDIOLA, F.; CODONY, R.; RAFECAS, M.; GRAU, A.; JORDÁN, A.; BOATELLA, J. Oxysterol formation in spray-dried egg processed and stored under various conditions: prevention and relationship with other quality parameters. J. Agric. Food Chem., Columbus, v.45, p.2229-2243, 1997.

HUBER, K.C.; PIKE, O.A.; HUBER, C.S. Antioxidant inhibition of cholesterol oxidation in a spray-dried food system during accelerated storage. J. Food Sci., Chicago, v.60, n. 5, p.909-916, 1995.

INSTITUTO ADOLFO LUTZ. Normas analiticas do Inst. Adolfo Lutz: métodos químicos e físicos para análises de alimentos. 3. ed., São Paulo: Instituto Adolfo Lutz, 1985.533p.

JIANG, Z:; SIM, J.S. Fatty acid modification of yolk lipids and cholesterol-lowering eggs. In: SIM, J.S.; NAKAI, S. eds. Egg uses and processing technologies - new developments. Wallingford: CAB International, 1996. p. 349-361.

LAI, S., GRAY, J.I., BUCKLEY, D.J., KELLY, P.M. Influence of free radicals and other factors on formation of cholesterol oxidation products in spray-dried whole egg. $J$. Agric. Food Chem., Columbus, v.43, p. 127-1131, 1995.

LERCKER, G.; RODRIGUEZ-ESTRADA, M.T., Cholesterol oxidation: presence of 7-ketocholesterol in different food products. J. Food Compos. Anal., Roma, v.13, p.625-631, 2000.

LI, S.X.; CHERIAN, G.; SIM, J.S. Cholesterol oxidation in egg yolk powder during storage and heating as affected by dietary oils and tocopherols. J. Food Sci., Chicago, v. 61, n. 4, p.721-725, 1996.

MAERKER, G., Cholesterol autoxidation current status. $J$. Am. Oil Chem. Soc., Champaign, v.64, n.3, p.388-392, 1987.

MAERKER, G; UNRUH JR, J. Cholesterol oxides I. Isolation and determination of some cholesterol oxidation products. J. Am. Oil Chem. Soc., Champaign, v.63, n.6, p.767-771, 1986.
MORALES-AIZPURÚA, I.C.;TENUTA-FILHO, A. Oxidation of cholesterol in mayonnaise during storage. Food Chem., Barking, v.89, p.611-615, 2005.

MORGAN, J.N.; ARMSTRONG, D.J. Quantification of cholesterol oxidation products in egg yolk powders spraydried with direct heating. J. Food Sci., Chicago, v.57, n.1, p.43-45, 1992.

NOUROOZ-ZADEH, J.; APPELQVIST, L.A. Cholesterol oxides in Swedish foods and food ingredients: fresh eggs and dehydrated egg products. J. Food Sci., Chicago, v.52, n.1, p.57-67, 1987.

OHTANI, K.; MIYABARA,K.; OKAMOTO, E.; KAMEL, M.; MATSUI-YUASA, I. Cytotoxicity of 7cetocholesterol toward cultured rat hepatocytes and the effect of vitamin E. Biosci. Biotechnol. Biochem., Tokyo, v.60, n.12, p. 989-1993, 1996.

OHTANI, K.; TERADA, E.; KAMEI, M.; MATSUI YUASA, I. Cytotoxicity of cholestane 3b,5a,6b-trol on cultured intestinal crypt cells (IEC-6). Biosci., Biotechnol. Biochem., Tokyo, v.61, n.4, p.573-576, 1997.

OSADA, K. Cholesterol oxidation products other biological effects. In: : GUARDIOLA, F. DUTTA, P.C., CODONY, R., SAVAGE, G.P. eds. Cholesterol and phytosterol oxidation products analysis, occurrence, and biological effects. Champaign: AOCS, 2002. cap.14, p.279-318.

PANIANGVAIT, P.; KING, A.J.; JONES, A.D.; GERMAN, B.G. Cholesterol oxides in foods of animal origin. J. Food Sci., Chicago, v.60, n.6, p.1159-1174, 1995.

PENAZZI, G.; CABONI, M. F.;ZUNIN, P.; EVANGELISTI, F.; TISCORNIA, E.; TOSCHI, T.G.; LERCKER, G. Routine high-performence liquid chromatographic determination of free 7-ketocholesterol in some foods by two different analytical methods. J. Am. Oil Chem. Soc., Champaign, v.72, n.2, p.1523-1527, 1995.

PENG, S.; HU, B.; MORIN,R.J. Angiotoxicity and atherogenicity of cholesterol oxides. J. Clin. Lab. Anal., New York, v.5, p.144-152, 1991.

PIE, J.E.; SPAHIS, K.; SEILLAN, C. Evaluation of oxidative degradation of cholesterol in food and food ingredients: identification and quantification of cholesterol oxides. $J$. Agric. Food Chem., Columbus, v.38, p.973-979, 1990. 
SANDER, B.D.; ADDIS, P.B.; PARK, S.W.; SMITH, D.E. Quantification of cholesterol oxidation products in a variety of foods. J. Food Prot., Ames, v. 52, n.2, p.109-114, 1989.

SARANTINOS, J.; O'DEA, K.; SINCLAIR, A.J. Cholesterol oxides in Australian foods identification and quantification. Food Aust., North Sydney, v.45, n.10, p. 485-490, 1993.

SEVANIAN, A.; PETERSON, A.R. The cytotoxic and mutagenic properties of cholesterol oxidation products. Food Chem. Toxicol., Oxford, v.24,n.10/11,p. 1103-1110, 1986.

SMITH, L.L., Cholesterol autoxidation 1981-1986. Chem. Phys. Lipid, Shannon, v.44, p.87-125, 1987.

SMITH, L.L.; JOHNSON, B.H. Biological activities of oxysterols. Free Radic. Biol. Med., New York, v.7, p.285-332, 1989.

TAI, C.Y.; CHEN, Y.C.; CHEN, B.H.Analysis, formation and inhibition of cholesterol oxidation products in foods: An overview (Part I). J. Food Drug Anal., Nankang, v.7, n.4, p.243-257, 1999.
TAI, C.Y.; CHEN, Y.C.; CHEN, B.H. Analysis, formation and inhibition of cholesterol oxidation products in foods: An overview (Part II). J. Food Drug Anal., Nankang, v.8, n.1,p.1-15, 2000.

TSAI, L.S.; HUDSON, C.A. Cholesterol oxides in commercial dry egg products: quantitation. J. Food Sci., Chicago, v.50, p.229-231, 1985.

VYNCKE, B.W. Direct determination of the thiobarbituric acid value in trichloracetic acid extracts of fish as a measure of oxidative rancidity. Fette Seifen Anstrichm., Leinfelden, v.72, n.12, p.1084-1087, 1970.

ZUNIN, P.; EVANGELISTI, F.; CABONI, M.F.; PENAZZI, G.; LERCKER, G.; TISCORNIA, E. Cholesterol oxidation in baked foods containing fresh and powdered eggs. J. Food Sci., Chicago, v.60, n.5, p.913916, 1995.

Recebido para publicação em 06 de dezembro de 2004. Aceito para publicação em 22 de março de 2005. 